\title{
The warm absorber in NGC 5548
}

\section{The lean years}

\author{
R. G. Detmers ${ }^{1}$, J. S. Kaastra ${ }^{1,2}$, E. Costantini ${ }^{1}$, I. M. McHardy ${ }^{3}$, and F. Verbunt ${ }^{2}$
}

\author{
1 SRON Netherlands Institute for Space Research, Sorbonnelaan 2, 3584 CA Utrecht, The Netherlands \\ e-mail: r.g.detmers@sron.nl \\ 2 Astronomical Institute, University of Utrecht, Postbus 80000, 3508 TA Utrecht, The Netherlands \\ 3 School of Physics and Astronomy, The University, Southampton SO17 1BJ, UK
}

Received 27 March 2008 / Accepted 16 April 2008

\section{ABSTRACT}

\begin{abstract}
We study the variability of the warm absorber and the gas responsible for the emission lines in the Seyfert 1 galaxy NGC 5548 to constrain the location and physical properties of these components. Using X-ray spectra acquired using the Chandra-LETGS in 2002 and 2005, we study the variability of the ionic column densities and line intensities. We measure a lower O vII forbidden emission line flux in 2005, while the $\mathrm{Fe} \mathrm{K} \alpha$ line flux remains constant. The warm absorber is less ionized in 2005, which enbles its location to be constrained to within $7 \mathrm{pc}$ of the central source. Using both the observed variability and the limit on the $F W H M$ of the O VII f line, we constrain the location of the narrow line region to a distance of $1 \mathrm{pc}$ from the central source. The apparent lack of variability in the Fe $\mathrm{K} \alpha$ line flux does enable a unique explanation to be derived.
\end{abstract}

Key words. galaxies: active - techniques: spectroscopic - galaxies: Seyfert - X-rays: individuals: NGC 5548

\section{Introduction}

Active Galactic Nuclei (AGN) have been studied for decades since their discovery over 60 years ago. Mass loss from the nucleus has been known for a long time to exist in the form of radio jets (see e.g. Peterson 1997) or in outflows showing broad absorption lines (Weymann et al. 1981). However, X-ray and UV observations have shown the presence of outflows in a majority of the moderately luminous Seyfert galaxies (Crenshaw et al. 1999). These outflows are important because they probe the inner regions of AGN and during the lifetime of an AGN they can produce a significant mass loss (Blustin et al. 2005). They can provide us with an understanding of the accretion process onto the supermassive black hole the enrichment of the intergalactic medium (Hamann \& Ferland 1999). The physical structure of these outflows and their connection to the other AGN components, such as the supermassive black hole, the accretion disk and the broad and narrow line region (BLR, NLR), is not understood. To learn more about these outflows and the other components of the AGN, variability studies are very important. They enable us to observe how the warm absorber and the emission lines respond to a variable flux from the central source. In addition they place constraints on the locations of the gas in which they originate, and provides us with information about their physical state.

A good object for the study of these processes is the Seyfert 1 galaxy NGC 5548. It is a relatively nearby AGN $(z=0.01676$, Crenshaw \& Kraemer 1999) and has a relatively high X-ray brightness. Previous UV and X-ray observations have shown the presence of a warm absorber in NGC 5548 (Steenbrugge et al. 2005; Crenshaw et al. 2003). From the kinematics it was concluded that the UV and X-ray absorption lines are part of the same outflow. By comparing a Chandra-HETGS and LETGS observation of NGC 5548 in 2002 with an earlier one in acquired
1999, Steenbrugge et al. (2005) conclude that there was no evidence for long-term variability, except for the $\mathrm{O} v$ absorption line strength.

We present results derived from a followup ChandraLETGS observation of NGC 5548 acquired in April 2005, which we compare with previous Chandra-LETGS and XMM-Newton RGS observations (Kaastra et al. 2002b; Steenbrugge et al. 2003, 2005). In all previously mentioned high resolution observations, the variability in the continuum flux was at most a factor of two and no significant variations were detected in the emission line strengths. During our new observation, the source was at a very low flux level from at least March 1 until May 10, as determined using Swift observations (Goad \& Page 2006), optical observations (Bentz et al. 2007), and this LETGS observation. During this time, the continuum flux was a factor five lower than in 2002. This enabled us to analyze the source for the first time at a very low flux level, and determine changes in spectral features, such as the warm absorber, narrow emission lines, and the iron $\mathrm{Fe} \mathrm{K} \alpha$ line. The Rossi X-ray Timing Explorer (RXTE) monitoring data of NGC 5548 from 1996 until 2007 are also presented to measure the long-term variability far more accurately than using only spectral observations, which have large gaps in coverage. We discuss the observation and data reduction in Sect. 2. Section 3 is used to describe the spectral analysis and the variability of the spectral components. We discuss our results in Sect. 4 and present our conclusions in Sect. 5.

\section{Data reduction}

NGC 5548 was observed for $141 \mathrm{ks}$ with the Chandra LETGS (Brinkman et al. 2000) on April 15 and 17 in 2005. The data were reduced as described by Kaastra et al. (2002b). In summary, the data were reduced using the standard CXC pipeline up until the level 1.5 event files. For the steps leading to the final 


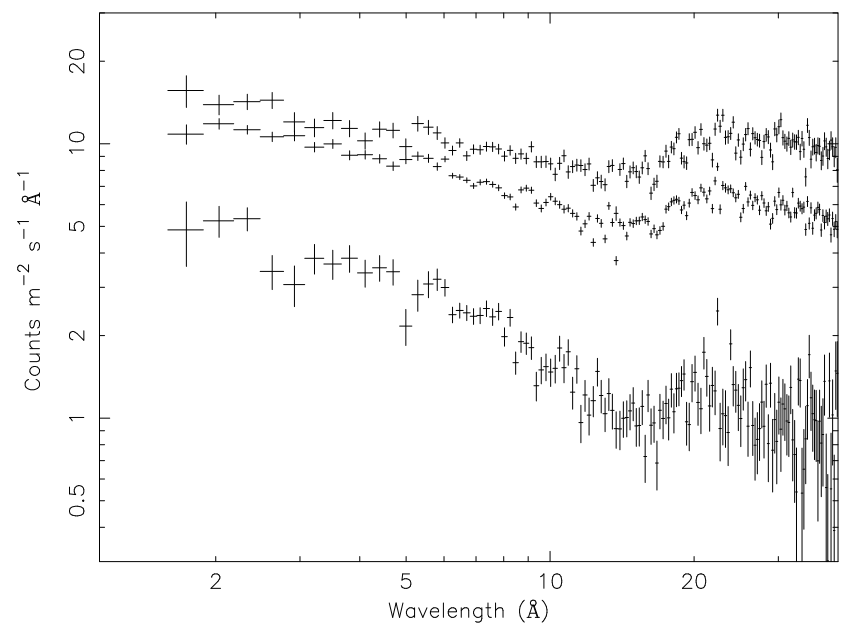

Fig. 1. The rebinned spectrum of NGC 5548 as taken with the LETGS instrument in 1999 (upper) 2002 (middle) and 2005 (lower).

event file, level 2, we followed an independent procedure, which is described in the aforementioned article. The resolution of the LETGS is $0.05 \AA$ and it spans a wavelength range of $1-180 \AA$. Because of the low signal to noise ratio and the Galactic absorption towards NGC 5548, we ignored the data above $80 \AA$ and below $1 \AA$. The spectrum was analyzed using the SPEX software package (Kaastra et al. 1996) ${ }^{1}$. We used $H_{0}=70 \mathrm{~km} \mathrm{~s}^{-1} \mathrm{Mpc}^{-1}$, $\Omega_{\mathrm{m}}=0.7$ and $\Omega_{\Lambda}=0.3$. Because of the low count rate we calculated the errors using C-statistics (see the XSPEC online man$\mathrm{ual}^{2}$; we use the form provided by Castor. See also Cash 1979). All errors indicated are $1 \sigma$ errors, computed for $\Delta C=1$. The RTXE data were reduced as described by McHardy et al. (2004).

\section{Spectral features}

Figure 1 presents the spectra from the 1999, 2002, and 2005 observations. The flux levels are much lower in the 2005 data, with a $2-10 \mathrm{keV}$ continuum flux of $1.47 \times 10^{-14} \mathrm{~W} \mathrm{~m}^{-2}$. To make sure that the spectral lines were analyzed using an adequate local continuum, we fitted the continuum with a spline model. A spline model enabled us to model the continuum between two boundaries $b_{1}$ and $b_{2}$ using a cubic spline. The limits were $1 \AA$ and $80 \AA$ in our case. The continuum flux $y_{i}$ is then given on 159 evenly spaced grid points $x_{i}$ between $b_{1}$ and $b_{2}$ with a spacing of $0.5 \AA$ between the grid points. The continuum model between the grid points was determined by cubic spline interpolation. The $y$ values of the grid points were dervied by spectral fitting. The use of a spline model implies that we did not assume an a priori shape for the continuum, for example a power law or black body continuum. We apply a redshift of 0.01676 to NGC 5548 and correct for Galactic extinction, where the H I column density was fixed to $1.65 \times 10^{24} \mathrm{~m}^{-2}$ (Nandra et al. 1993). We add various absorption components and narrow emission lines to this continuum (see Sects. 3.1-3.3). The best fit (including all of the features) has $C=3228$ for 2833 degrees of freedom.

\footnotetext{
1 See also http://www.sron.nl/spex

2 http://heasarc.gsfc.nasa.gov/docs/xanadu/xspec/ manual/XSappendixCash.html
}

Table 1. Oxygen column densities for the 2005 observation. The logarithms of the column densities are listed in $\mathrm{m}^{-2}$.

\begin{tabular}{lllll}
\hline \hline$v\left(\mathrm{~km} \mathrm{~s}^{-1}\right)$ & -1040 & -530 & -160 & Total \\
$\sigma\left(\mathrm{km} \mathrm{s}^{-1}\right)$ & $127 \pm 28$ & $62 \pm 21$ & $120 \pm 34$ & \\
Ion & & & & \\
\hline O III & $<20.5$ & $<20.6$ & $<21.2$ & $<21.4$ \\
O IV & $20.8 \pm 0.5$ & $<20.9$ & $21.0 \pm 0.5$ & $21.2 \pm 0.5$ \\
O v & $20.9 \pm 0.6$ & $<21.3$ & $21.4 \pm 0.5$ & $21.5 \pm 0.5$ \\
O VI & $22.1 \pm 0.2$ & $<20.9$ & $<21.4$ & $22.1 \pm 0.2$ \\
O VII & $<21.0$ & $<20.4$ & $21.1 \pm 0.5$ & $<21.6$ \\
O VIII & $<21.1$ & $<21.7$ & $21.2 \pm 0.5$ & $<21.9$ \\
\hline$\sigma\left(\mathrm{km} \mathrm{s}^{-1}\right.$, fixed $)$ & 40 & 100 & 90 & \\
\hline O III & $<20.4$ & $<20.4$ & $<21.1$ & $<21.2$ \\
O IV & $20.6 \pm 0.6$ & $<21.0$ & $21.1 \pm 0.4$ & $21.3 \pm 0.3$ \\
O V & $21.3 \pm 0.4$ & $20.5 \pm 0.7$ & $21.4 \pm 0.3$ & $21.7 \pm 0.2$ \\
O VI & $21.9 \pm 0.2$ & $<21.3$ & $21.1 \pm 0.4$ & $22.0 \pm 0.2$ \\
O VII & $21.5 \pm 0.9$ & $<20.6$ & $21.0 \pm 0.5$ & $21.6 \pm 0.5$ \\
O VIII & $<21.5$ & $<21.5$ & $21.1 \pm 0.9$ & $21.3 \pm 0.6$ \\
\hline
\end{tabular}

\subsection{Warm absorber}

To model the warm absorber, we use the slab model in SPEX (Kaastra et al. 2002a), which calculates the transmission of a thin, irradiated slab of matter with a set of adjustable ionic column densities. We model the optical depth of the absorption lines using Voigt profiles. Our models consist of 3 slab components, for which the ionic column densities are allowed to vary and the outflow velocities $v$ fixed to the values given in the previous LETGS study (Steenbrugge et al. 2005). Simultaneous UV observations of NGC 5548 (Crenshaw et al. 2003) provide evidence for five different warm absorber outflow velocities, but due to the lower spectral resolution in the X-ray, only three outflow velocities could be measured reliably by Steenbrugge et al. (2005), namely $-1040,-530$ and $-160 \mathrm{~km} \mathrm{~s}^{-1}$.

A change in the optical depth of an absorption line can be due to a change in the velocity broadening $\sigma$ or a change in the ionic column density $N_{\text {ion }}$. Alternatvely a change in the covering factor of the absorbing gas can also explain the variations in the absorption lines. We assume a covering factor of unity for the absorbing gas (see Sect. 4 for discussion). To be certain that the changes we observe in the absorption lines are indeed due to column density changes and not only due to a possible change in $\sigma$, we performed two fits, one in which $\sigma$ was allowed to vary, the other in which $\sigma$ was fixed to the values reported by Steenbrugge et al. (2005). Figure 2 shows the 2002 and 2005 total column densities for both models. The O VIII column density has decreased, while the O IV-O VI column densities have increased. The total column densities are similar for both models. In Table 1, we display the 2005 oxygen column densities for both models.

\subsection{Narrow emission lines}

The O VII forbidden line at $22.101 \AA$ is clearly visible in the spectrum of the 2005 observation. We also searched for the Ne IX forbidden line at $13.699 \AA$, since that line was also detected in earlier observations. Table 2 indicates the strengths of both lines in the new spectrum and in earlier observations. We measure a flux of $0.35 \pm 0.06 \mathrm{ph} \mathrm{m}^{-2} \mathrm{~s}^{-1}$ for the $\mathrm{O}$ VII forbidden emission line and detect a blueshift of $-250 \pm 70 \mathrm{~km} \mathrm{~s}^{-1}$ for the O VII $f$ line. For the Ne IX forbidden emission line, we measure an upper limit of $0.04 \mathrm{ph} \mathrm{m}^{-2} \mathrm{~s}^{-1}$, if we assume that the Ne IX line has the same blueshift as the O VII $f$ line. Earlier 


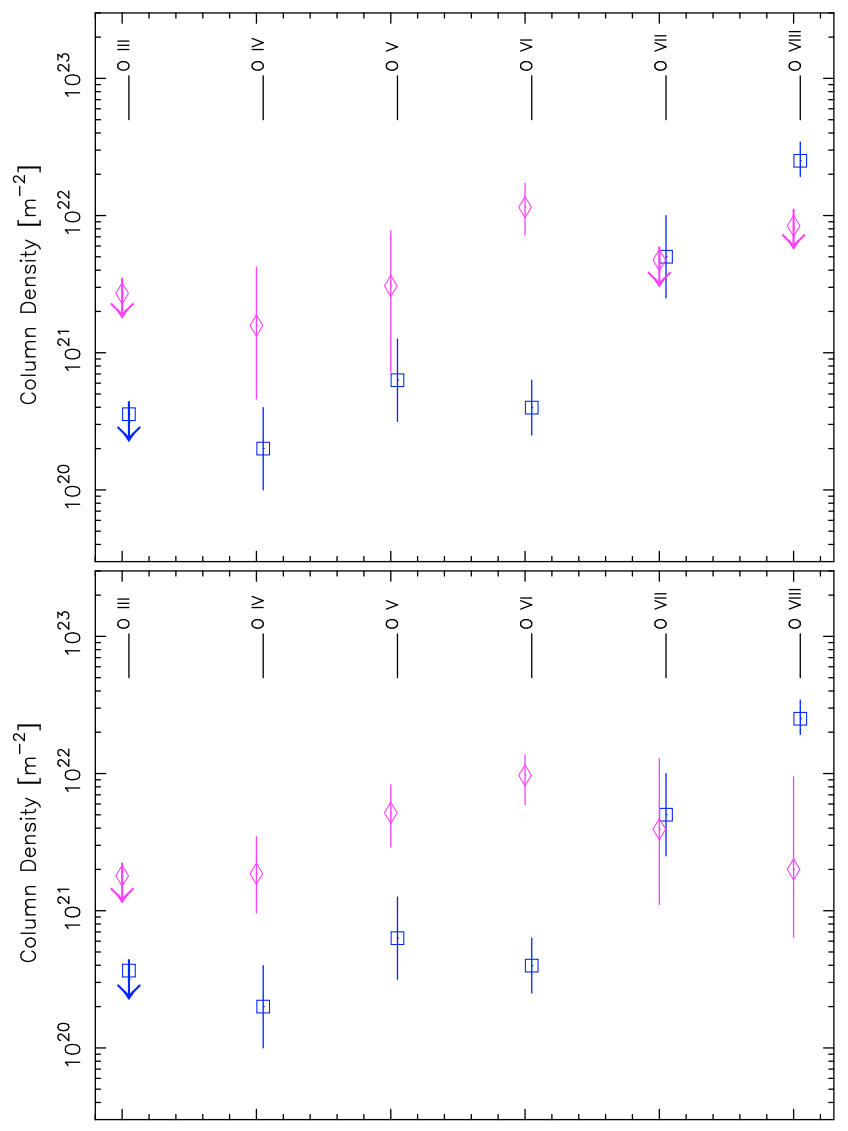

Fig. 2. Total oxygen column densities as measured with the LETGS instrument in 2002 (rectangles) and 2005 (diamonds). $1 \sigma$ errors are shown as well, apart from ions for which an upper limit to the total column density can be measured (arrows). The upper panel shows the total column densities for which the velocity broadening $\sigma$ was allowed to vary, the lower panel where $\sigma$ was fixed to the values of Steenbrugge et al. (2005).

Table 2. The unabsorbed flux in $\mathrm{ph}^{-2} \mathrm{~s}^{-1}$ for the O VII and Ne IX forbidden emission lines for all high resolution spectral observations of NGC 5548. All errors are calculated at a $68 \%$ confidence level.

\begin{tabular}{lcccc}
\hline \hline Year (month) & Instrument & O VII & Ne IX & Reference $^{a}$ \\
\hline 1999 (December) & LETGS & $0.81 \pm 0.13$ & $0.25 \pm 0.07$ & 1 \\
2000 (February) & MEG & $0.82 \pm 0.18$ & $0.09 \pm 0.03$ & 1 \\
2000 (December) & RGS & $0.65 \pm 0.18$ & $0.14 \pm 0.07$ & 2 \\
2001 (July) & RGS & $0.70 \pm 0.16$ & $0.22 \pm 0.07$ & 2 \\
2002 (January) & LETGS & $0.88 \pm 0.08$ & $0.14 \pm 0.04$ & 3 \\
2005 (April) & LETGS & $0.35 \pm 0.06$ & $<0.04$ & 4 \\
\hline
\end{tabular}

${ }^{a}$ References for previous observations: (1) Kaastra et al. (2002b); (2) extracted from the public archive, errors include uncertainty due to continuum level; (3) Steenbrugge et al. (2005); (4) present work.

observations, as described in Sect. 3 reported blueshifts of $-150 \pm 70 \mathrm{~km} \mathrm{~s}^{-1}$ (Steenbrugge et al. 2005, HEG) and $-70 \pm$ $100 \mathrm{~km} \mathrm{~s}^{-1}$ (Kaastra et al. 2002b). The LETGS has a wavelength scale uncertainty of $0.01 \AA$, or $140 \mathrm{~km} \mathrm{~s}^{-1}$ at the O VII f emission line. Taking this and the error on the outflow velocity into account, the blueshift that we detect is negligible, but it is however consistent with the HEG measurement (Steenbrugge et al. 2005). The decrease in flux with respect to previous observations is consistent for both lines, although we are only able to measure an upper limit for the Ne IX $\mathrm{f}$ line.

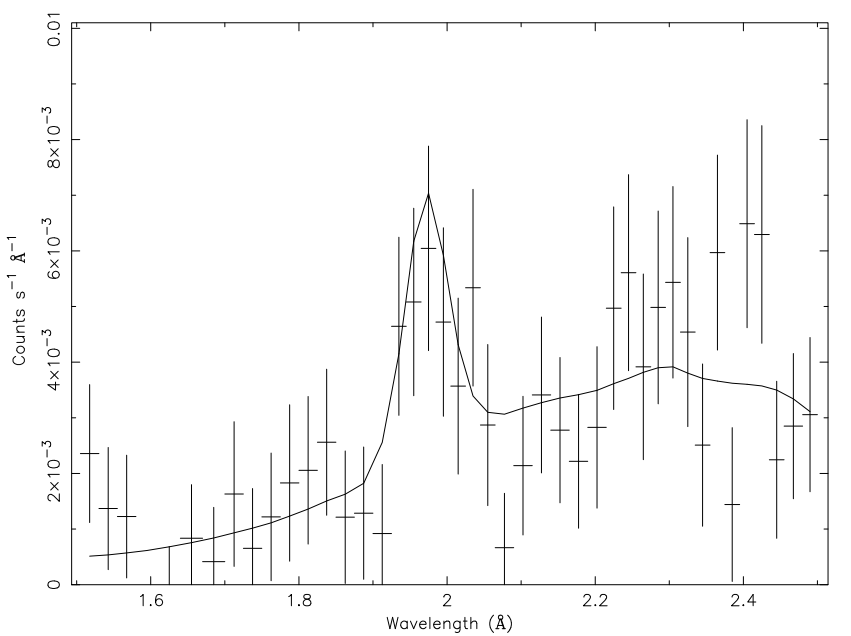

Fig. 3. The $\mathrm{Fe} \mathrm{K} \alpha$ emission line fit for the 2005 observation.

Table 3. Fe $\mathrm{K} \alpha$ line parameters. All errors are calculated with $68 \%$ confidence.

\begin{tabular}{lcccc}
\hline \hline & HEG(2000) & EPIC(2001) & HEG(2002) & LETGS(2005) \\
\hline$E(\mathrm{keV})$ & $6.40 \pm 0.02$ & $6.39 \pm 0.01$ & $6.39 \pm 0.01$ & $6.39 \pm 0.03$ \\
$E W(\mathrm{eV})$ & $130 \pm 30$ & $60 \pm 9$ & $47 \pm 11$ & $420 \pm 140$ \\
$F W H M\left(\mathrm{~km} \mathrm{~s}^{-1}\right)$ & $4500 \pm 1600$ & $6500 \pm 1300$ & $1700 \pm 1100$ & $7300 \pm 5100$ \\
Flux $\left(\mathrm{ph} \mathrm{m}^{-2} \mathrm{~s}^{-1}\right)$ & $0.36 \pm 0.10$ & $0.38 \pm 0.06$ & $0.24 \pm 0.06$ & $0.55 \pm 0.18$ \\
Reference $^{a}$ & 1 & 2 & 3 & 4 \\
\hline
\end{tabular}

${ }^{a}$ References for previous Fe K $\alpha$ detections: (1) Yaqoob et al. (2001); (2) Pounds et al. (2003); (3) Steenbrugge et al. (2005); (4) present work.

\subsection{The narrow Fe Ko line}

The low continuum flux facilitates the detection of a clear $\mathrm{Fe} \mathrm{K} \alpha$ emission line at $6.39 \pm 0.03 \mathrm{keV}$ in the spectrum of our LETGS data (Fig. 3). Using a Gaussian fit, we measure a flux of $0.55 \pm 0.18 \mathrm{ph} \mathrm{m}^{-2} \mathrm{~s}^{-1}$, which agrees with previous values from Yaqoob et al. (2001), Pounds et al. (2003), and Steenbrugge et al. (2005) as shown in Table 3. The equivalent width of the line is $420 \pm 140 \mathrm{eV}$, much larger than measured in those previous observations. There is no evidence for a relativistically broadened $\mathrm{Fe} \mathrm{K} \alpha$ emission line profile in our spectrum. We measure a $F W H M$ of $7300 \pm 5100 \mathrm{~km} \mathrm{~s}^{-1}$, which agrees with previous measurements.

\section{Discussion}

\subsection{The location of the warm absorber}

Figure 2 shows that the O VIII column density has decreased, while the O IV-O VI column densities have increased between 2002 and 2005, both for $\sigma$ fixed and $\sigma$ set free. Some errors in the oxygen column densities in the 2005 spectrum are large, due to a combination of the low statistics and saturation of some of the absorption lines. As shown in Fig. 4 there are additional indications that the column density of the lower ionized oxygen ions has increased from 2002 to 2005. The O V absorption line at $22.354 \AA$ is indeed much stronger and broader than in either 1999 or 2002 . The equivalent width of the absorption line is $140 \pm 30 \mathrm{~m} \AA$, compared to $44 \pm 6 \mathrm{~m} \AA$ in 2002 and $22 \pm 9 \mathrm{~m} \AA$ in 1999. Allowing the velocity width $\sigma$ to vary, the column densities of the individual velocity components do change, but the total column densities do not change significantly (see Table 1). This is a further indication that the observed changes in the 


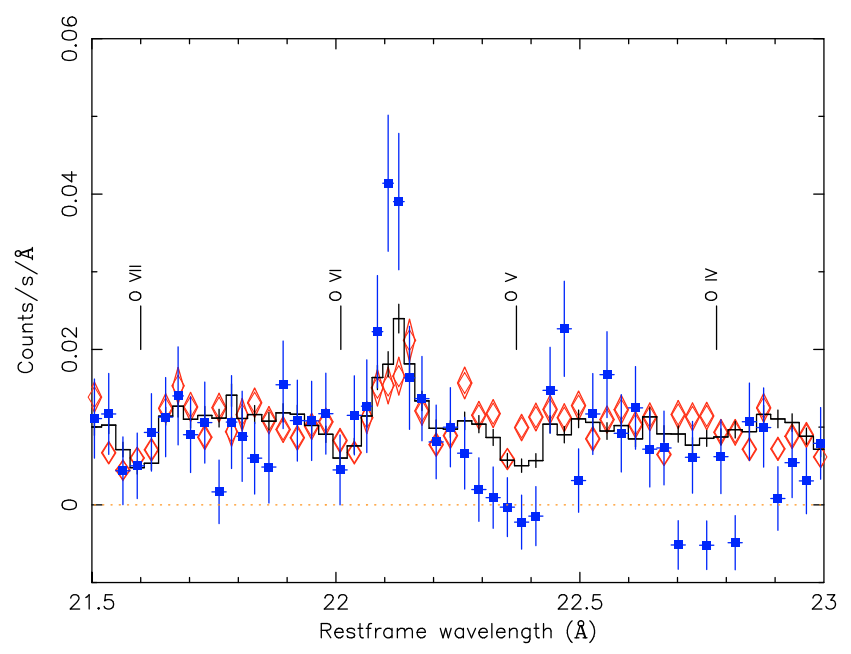

Fig. 4. Comparison of the 1999 (diamonds), 2002 (solid line) and 2005 (rectangles) spectra, close to the forbidden O VII emission line at $22.101 \AA$. The deep O V absorption line is visible at $22.354 \AA$. The 1999 and 2005 spectra have been scaled to the 2002 flux.

absorption lines are indeed due to variations in the column density and not only due to a possible change in $\sigma$.

In principle, this still leaves open the possibility of a varying covering factor of the X-ray absorbing gas. As shown in UV spectral observations of NGC 5548 (Crenshaw et al. 2003), the covering factor for the various components is not unity. In the $\mathrm{UV}$, it is possible to resolve the different velocity components of the absorber, which is difficult, if not impossible to achieve at the resolution of current X-ray instruments. The size of the $\mathrm{X}$-ray emitting region $\left(10 R_{\mathrm{g}}\right.$ is estimated to be far smaller than the size of the UV emitting region. Also, the BLR region is measured to be larger in the optical/UV than in the X-rays (see e.g. Costantini et al. 2007). This, and the fact that column densities for the highly ionized oxygen species have decreased, while column densities of the lowly ionized species have increased, allows us to be confident that the assumption of a covering factor of unity for the X-ray absorbing gas is an accurate one. Therefore the large increase in O VI can be explained by recombination of $\mathrm{O}$ VIII and $\mathrm{O}$ VII.

The ionization parameter $\xi$ is determined by the ratio of the ionizing flux and the density of the gas (Tarter et al. 1969):

$\xi=\frac{L}{n R^{2}}$.

Here $L$ is the $1-1000$ Rydberg luminosity, $n$ is the density of the gas and $r$ is the distance from the ionizing source. We are only able to determine the product of the density and distance squared, $n R^{2}$, from the luminosity and $\xi$. The luminosity $L=$ $9.0 \times 10^{36} \mathrm{~W}$ was measured for the 2002 LETGS observation (Steenbrugge et al. 2005). We first consider changes in the O VIII column density to obtain a lower limit for the density $n$. The column density of O VIII in the 2002 LETGS observation is $3.39 \times 10^{22}$ (See model B, Steenbrugge et al. 2005). We assume that the value for the ionization parameter is the one at which the column density of the ion peaks, such that $\log \xi=1.65$ for O VIII, using the XSTAR code ${ }^{3}$ for photoionization modeling. For determining $n$, we assume that the changes that we detect are due to recombination. The density $n$ scales inversely with the recombination time $\tau_{\text {rec }}$ of the gas and depends on the

${ }^{3}$ http://heasarc.gsfc.nasa.gov/xstar/xstar.html recombination rate of the specific ion (Krolik \& Kriss 1995; Bottorff et al. 2000):

$\tau_{\mathrm{rec}}\left(X_{i}\right)=\left(\alpha_{\mathrm{r}}\left(X_{i}\right) n\left[\frac{f\left(X_{i+1}\right)}{f\left(X_{i}\right)}-\frac{\alpha_{\mathrm{r}}\left(X_{i-1}\right)}{\alpha_{\mathrm{r}}\left(X_{i}\right)}\right]\right)^{-1}$,

where $\alpha_{\mathrm{r}}\left(X_{i}\right)$ is the recombination rate from ion $X_{i=1}$ to ion $X_{i}$ and $f\left(X_{i}\right)$ is the fraction of element $X$ in ionization state $i$. If $\tau_{\text {rec }}$ is positive, it implies that the amount of O VIII increases, by recombination of O IX. If $\tau_{\text {rec }}$ is negative, then this means that more $\mathrm{O}$ VIII is destroyed by recombination to $\mathrm{O}$ VII than created by recombination of O IX.

Since the change in column densities occurred between Jan. 21, 2002 and April 15 2005, the upper limit to $\tau_{\text {rec }}$ is 1160 days (in the rest frame of the source). The $f\left(X_{i+1}\right)$ to $f\left(X_{i}\right)$ for $i=\mathrm{O}$ VIII ratio can be determined from the ionization parameter $\xi$. For $\log \xi=1.65$, the fraction of O IX to O VIII is 0.48 . From $\xi$ we derive a temperature of $86000 \mathrm{~K}$. With this value of $T, \alpha_{\mathrm{r}}(\mathrm{O}$ VII $)=8.65 \times 10^{-18} \mathrm{~m}^{3} \mathrm{~s}^{-1}$ and $\alpha_{\mathrm{r}}(\mathrm{O}$ VIII $)=$ $1.31 \times 10^{-17} \mathrm{~m}^{3} \mathrm{~s}^{-1}$ (Nahar 1999). Using these values, we find a lower limit to the density of $4.2 \times 10^{9} \mathrm{~m}^{-3}$. Using this limit for $n$ and (1), we get an upper limit to the distance of $R<7$ pc. If we instead use $\mathrm{O} v$ to determine the distance, using $\log \xi=-0.2$ and $T=19000 \mathrm{~K}$, we derive an upper limit of $R<19 \mathrm{pc}$ The main uncertainty causing the large upper limits is of course the large gap in the spectral data between 2002 and 2005.

\subsection{The variable $O \mathrm{vII} f$ narrow emission line}

The line flux of the $\mathrm{O}$ VII $\mathrm{f}$ line has decreased from $0.88 \pm 0.12$ to $0.35 \pm 0.06 \mathrm{ph} \mathrm{m}^{-2} \mathrm{~s}^{-1}$ between 2002 and 2005 (Table 2). This immediately provides us an upper limit to the distance, namely the light travel distance of $1 \mathrm{pc}$. A lower limit to the distance of the NLR gas can be obtained from the velocity dispersion and the mass of the supermassive black hole as shown by Netzer (1990):

$$
M=\frac{3 r V_{F W H M}^{2}}{4 G}
$$

where $M$ is the mass of the supermassive black hole, $V_{F W H M}$ is the $F W H M$ of the line and $r$ is the distance of the line emitting region. This assumes an isotropic velocity distribution, which is reasonable, since we cannot obtain any detailed line profile information for the NLR region. Using $M=(6.54 \pm 0.26) \times$ $10^{7} M_{\odot}$ (Bentz et al. 2007) and an upper limit to $V_{F W H M}$ of $560 \mathrm{~km} \mathrm{~s}^{-1}$ obtained by using a Gaussian line model for the O VII $f$ line instead of a delta line, we obtain a lower limit of $1.2 \mathrm{pc}$ for the location of the NLR. If the NLR instead has the same velocity distribution as the BLR in AGN (Peterson et al. 2004), then the lower limit would be 0.9 pc. A similar limit of $\sim 1$ pc was also derived by Kaastra et al. (2003). Although this lower limit can vary by a factor of a few, depending on the exact velocity distribution and the upper limit can be smaller as it is based on sparse temporal sampling, both limits used constrain the location of the NLR to be about $1 \mathrm{pc}$. This is the first time that a well-constrained location has been determined, using the combination of variability and line width limits. Optical measurements (Kraemer et al. 1998) indicate that the optical narrow line emission originates in a region within $70 \mathrm{pc}$, with a high ionization component at $1 \mathrm{pc}$, in agreement with our findings.

We have ascertained whether $\tau_{\text {rec }}$ was much smaller than $\tau_{\text {lt }}$, so that the total delay is determined only by $\tau_{\mathrm{lt}}$ :

$\tau_{\text {delay }}=\tau_{\text {lt }}+\tau_{\text {rec }}$, 
where $\tau_{\text {delay }}$ is the total delay time between changes in the continuum flux and changes in the emission line flux, $\tau_{\mathrm{lt}}$ is the light travel time through the emitting region, and $\tau_{\text {rec }}$ is the recombination timescale. Using Eq. (1) with $\log \xi=1$ (where the O VII fraction in the gas peaks) and $R=1 \mathrm{pc}$, we dervie a lower limit to $n$ of $1 \times 10^{12} \mathrm{~m}^{-3}$. From $\xi$ we can determine the temperature for the gas with $\log \xi=1$, which is $33000 \mathrm{~K}$. The fraction $\mathrm{O}$ VIII/O VII $=0.25$ for $\xi=1$.

Using the O VII and O VI recombination rates from Nahar $\left(1999, \alpha_{\mathrm{r}}(\mathrm{O} \mathrm{VII})=1.65 \times 10^{-17} \mathrm{~m}^{3} \mathrm{~s}^{-1}\right.$ and $\alpha_{\mathrm{r}}(\mathrm{O} \mathrm{VI})=0.98 \times$ $10^{-17} \mathrm{~m}^{3} \mathrm{~s}^{-1}$ ), the upper limit to $\tau_{\text {rec }}$ is 3 days, far less than the 1160 days limit from variability arguments. This implies that the total delay time $\tau_{\text {delay }}$ is dependent only on the light travel time $\tau_{\mathrm{lt}}$.

\subsection{The origin of the $\mathrm{Fe} \mathrm{K} \alpha$ line}

Due to the low continuum flux, we are able to detect the Fe $\mathrm{K} \alpha$ emission line using the LETGS data, even though the LETGS is not optimized for the wavelength range where the line is present, since its spectral resolution is $50 \mathrm{~m} \AA$. The interesting result is that the line flux is in agreement with earlier observations, even though we would have expected a decrease in line flux if the line flux correlates with the continuum flux. Because of the low continuum flux and the constant line flux the equivalent width $(E W)$ of the line is large, $420 \pm 140 \mathrm{eV}$.

Using both HEG measurements of the Fe K $\alpha$ line (Table 3), the weighted average of the $F W H M$ is $2600 \pm 900 \mathrm{~km} \mathrm{~s}^{-1}$. Once again using Eq. (3), the line is formed at $0.06 \pm 0.04 \mathrm{pc}$ from the central source, assuming again a mass of $\sim 6.54 \times 10^{7} \mathrm{M}_{\odot}$. At this distance, one would expect that the iron line would also have responded to the lower ionizing flux, since the light travel time is far less than 3 years.

There are at least five possible explanations for the constant flux and corresponding larger $E W$ of the emission line. The first one that can be immediately dismissed is the light-bending close to a black hole (Miniutti \& Fabian 2004). The derived distance from the $F W H M$ of the line $\left(0.06 \mathrm{pc}\right.$, or $\left.19000 R_{\mathrm{g}}\right)$ is much too large for any light-bending to play a role.

The second reason why the iron line is unaffected by the lower continuum could be that the line consists of multiple unresolved components originating in a broad range of distances from the central source. If we assume that this is the case, then at least part of the emission could originate from a region further away from the central source than $1 \mathrm{pc}$, for instance the torus structure on a parsec-scale (Antonucci 1993). However, Suganuma et al. (2006) performed infrared reverberation mapping of NGC 5548 and found a time-lag of 47-53 days between the continuum flux in the $V$ band and the IR emission (dust). This would place the dust at $\sim 0.04 \mathrm{pc}$. The dust may of course extent much further outward; so the possibility that Fe $\mathrm{K}$ emission is produced by reflection on dust further than $1 \mathrm{pc}$ away from the central source should therefore be considered. However, if the bulk of the $\mathrm{Fe} \mathrm{K} \alpha$ line originated in a source of size close to the pc-scale, the line would be far narrower than observed.

Another explanation is that the continuum was affected by a peak in flux just before our observation was acquired, such that the $\mathrm{Fe} \mathrm{K} \alpha$ line had already responded to this increase in continuum flux, but the warm absorber and the narrow emission lines had not. From the LETGS observation alone this cannot be ruled out, although optical observations were also acquired (March 1 till April 10, Bentz et al. 2007) for NGC 5548 just before the LETGS observation. They detected a very low

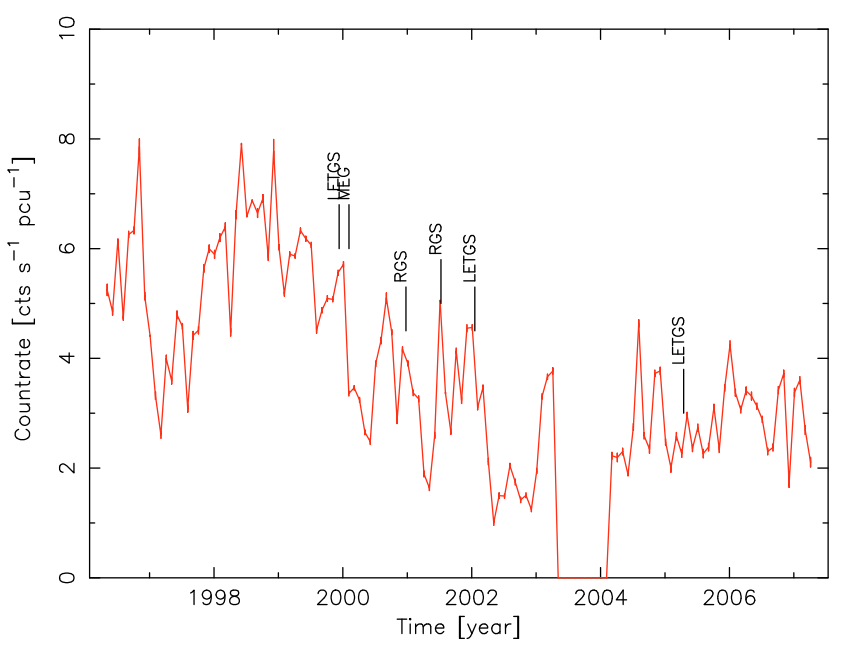

Fig. 5. RXTE lightcurve of NGC 5548, rebinned to one month intervals, spanning from 1996 till 2007. The count rate shown is per PCU detector of the RXTE. All 6 spectral observations (see Table 2) are shown as well. There is a gap in the data from the beginning of 2003 till 2004, when the source was not monitored by RXTE. The errors in the countrates are at the $1 \%$ level.

continuum and a weaker $\mathrm{H} \beta$ emission line. Since the $\mathrm{H} \beta$ line has a time-lag of 6.3 days, any peak in continuum flux should have been evident during the analysis of the optical observations. The RXTE monitoring also did not detect a peak in the months prior to our observation.

The fourth possibility is related to the variation in continuum flux and its effect on the gas, which is studied using the Fe K line. Matt et al. (1993) demonstrated that the strength of the $\mathrm{Fe} \mathrm{K} \alpha$ line depends on the ionization parameter $\xi$ of the reflecting gas. They showed that if $\xi \leq 100$, the line strength increases as $\xi$ decreases. Since the continuum flux has decreased suddenly, it is not unreasonable to assume that the ionization state of the gas producing the $\mathrm{Fe} \mathrm{K} \alpha$ line will be lower. This implies that the line flux could remain constant while the continuum flux drops.

The final possibility is that, while the continuum flux in the 2-10 keV band has decreased dramatically, the reflection component above $10 \mathrm{keV}$, which produces the $\mathrm{Fe} \mathrm{K} \alpha$ line, may have been far less variable than the continuum. This behavior was in fact detected in several AGN with Suzaku (Reeves et al. 2006). The reason for the lack of strong variability in the reflection component is still unknown. Due to the errors in the $\mathrm{Fe} \mathrm{K} \alpha$ line flux and the instrumental resolution of the LETGS, we are, however, unable to distinguish among these explanations.

\subsection{Constraints from RXTE monitoring}

Figure 5 shows the long timescale light curve of NGC 5548 as observed by RXTE from 1996 until 2007. The data have been rebinned into time intervals of one month. As can be seen, the variability on a timescale of a few months can be larger than on longer timescales of years. Long-term changes are still visible. From 2002 onward, the average count rate is 20-25\% lower than that of the earlier years. Applying the information we have on the continuum flux of NGC 5548 between 2002 and 2005, we can refine our estimate of the upper limit to the recombination timescale of the warm absorber.

For an upper limit to the variability timescale of the warm absorber, we currently use an upper limit of 1160 days. Based only on the analysis of two LETGS spectra, this timescale is 
uncertain. We note that the RXTE continuum flux was at approximately the same level in mid-2004 as during the 2002 LETGS observation. If the ionization timescale is short, the ionization state in mid-2004 might have been the same to that in 2002; in which case a more accurate estimate for the recombination timescale may be one year, and even that may be far too long. There is a peak in the X-ray flux only a few months before our 2005 observation, so an upper limit of a few months may be even a more accurate estimate indeed. If the recombination timescale is 4 months instead of 1160 days, the upper limit to the warm absorber distance would instead be 2 pc. Since the recombination timescale is so uncertain and probably seriously overestimated, we therefore conclude that the warm absorber is probably much further in than the $7 \mathrm{pc}$ we derived as an upper limit. Further studies, taking time-dependent ionization into account, will enable the changes in the warm absorber to be modeled more accurately as it reacts to the continuum variations observed by RXTE.

Concerning the changes in the emission lines, previous observations have measured no observable change in flux for both lines (see Tables 2 and 3), even though the continuum varied substantially between those observations. This is a clear indication that both emission lines respond to the continuum changes on a long timescale, of years not weeks or months. For the Fe K $\alpha$ line this would imply that any correlation between the continuum flux and the line flux is more complex than a linear relation between the two. Possible correlations between the emission lines and continuum flux will be investigated further in a future paper.

\section{Conclusions}

This is the first detailed X-ray analysis of NGC 5548 at an extremely low flux level. The warm absorber, which showed little signs of change in previous observations, shows clear signs of recombination to a lower ionized state. We have constrained its location to within $7 \mathrm{pc}$ of the central source, although this is most likely a serious overestimate. The narrow forbidden $\mathrm{O}$ VII line has decreased in flux, which along with the limit on the line width, places its location at $\sim 1 \mathrm{pc}$ from the central source. This location also agrees with previous estimates for this source. Only the $\mathrm{Fe} \mathrm{K} \alpha$ emission line has not responded linearly to the lower continuum flux, the line flux is consistent with earlier observations. The most likely explanations are a change in the ionization state of the reflecting gas or a constant reflection component that is not related closely to the $2-10 \mathrm{keV}$ continuum.
Acknowledgements. SRON is supported financially by NWO, The Netherlands Organization for Scientific Research.

\section{References}

Antonucci, R. 1993, ARA\&A, 31, 473

Bentz, M. C., Denney, K. D., Cackett, E. M., et al. 2007, ApJ, 662, 205

Blustin, A. J., Page, M. J., Fuerst, S. V., Branduardi-Raymont, G., \& Ashton, C. E. 2005, A\&A, 431, 111

Bottorff, M. C., Korista, K. T., \& Shlosman, I. 2000, ApJ, 537, 134

Brinkman, A. C., Gunsing, C. J. T., Kaastra, J. S., et al. 2000, ApJ, 530, L111

Cash, W. 1979, ApJ, 228, 939

Costantini, E., Kaastra, J. S., Arav, N., et al. 2007, A\&A, 461, 121

Crenshaw, D. M., \& Kraemer, S. B. 1999, ApJ, 521, 572

Crenshaw, D. M., Kraemer, S. B., Boggess, A., et al. 1999, ApJ, 516, 750

Crenshaw, D. M., Kraemer, S. B., Gabel, J. R., et al. 2003, ApJ, 594, 116

Goad, M. R., \& Page, K. L. 2006, in Proceedings of the The X-ray Universe 2005, ed. A. Wilson, ESA SP-604, 623

Hamann, F., \& Ferland, G. 1999, ARA\&A, 37, 487

Kaastra, J. S., Mewe, R., \& Nieuwenhuijzen, H. 1996, in UV and X-ray Spectroscopy of Astrophysical and Laboratory Plasmas (Tokyo: Universal Academy Press), ed. K. Yamashita, \& T. Watanabe, Frontiers Science Series, 15,411

Kaastra, J. S., Steenbrugge, K. C., Brinkman, A. C., \& Edelson, R. 2002a, in X-ray Spectroscopy of AGN with Chandra and XMM-Newton, ed. T. Boller, S. Komossa, S. Kahn, H. Kunieda, \& L. Gallo, 15

Kaastra, J. S., Steenbrugge, K. C., Raassen, A. J. J., et al. 2002b, A\&A, 386, 427 Kaastra, J. S., Steenbrugge, K. C., Brinkman, A. C., et al. 2003, in Active Galactic Nuclei: From Central Engine to Host Galaxy, ed. S. Collin, F. Combes, \& I. Shlosman, ASP Conf. Ser., 290, 101

Kraemer, S. B., Crenshaw, D. M., Filippenko, A. V., \& Peterson, B. M. 1998, ApJ, 499, 719

Krolik, J. H., \& Kriss, G. A. 1995, ApJ, 447, 512

Matt, G., Fabian, A. C., \& Ross, R. R. 1993, MNRAS, 262, 179

McHardy, I. M., Papadakis, I. E., Uttley, P., Page, M. J., \& Mason, K. O. 2004, MNRAS, 348, 783

Miniutti, G., \& Fabian, A. C. 2004, MNRAS, 349, 1435

Nahar, S. N. 1999, ApJS, 120, 131

Nandra, K., Fabian, A. C., George, I. M., et al. 1993, MNRAS, 260, 504

Netzer, H. 1990, in 20 Saas-Fee Advanced Course of the Swiss Society for Astrophysics and Astronomy: Active galactic nuclei, ed. R. D. Blandford, H. Netzer, L. Woltjer, T. J.-L. Courvoisier, \& M. Mayor, 57

Peterson, B. M. 1997, An Introduction to Active Galactic Nuclei (Cambridge, New York: Cambridge University Press), Physical description XVI, 238 Peterson, B. M., Ferrarese, L., Gilbert, K. M., et al. 2004, ApJ, 613, 682 Pounds, K. A., Reeves, J. N., Page, K. L., et al. 2003, MNRAS, 341, 953 Reeves, J. N., Fabian, A. C., Kataoka, J., et al. 2006, Astron. Nachr., 327, 1079 Steenbrugge, K. C., Kaastra, J. S., de Vries, C. P., \& Edelson, R. 2003, A\&A, 402, 477

Steenbrugge, K. C., Kaastra, J. S., Crenshaw, D. M., et al. 2005, A\&A, 434, 569 Suganuma, M., Yoshii, Y., Kobayashi, Y., et al. 2006, ApJ, 639, 46 Tarter, C. B., Tucker, W. H., \& Salpeter, E. E. 1969, ApJ, 156, 943 Weymann, R. J., Carswell, R. F., \& Smith, M. G. 1981, ARA\&A, 19, 41 Yaqoob, T., George, I. M., Nandra, K., et al. 2001, ApJ, 546, 759 